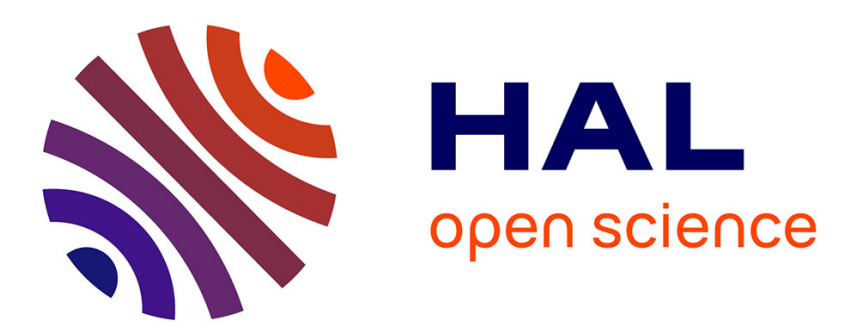

\title{
Modeling Viscoelastic Properties of Loudspeaker Suspensions Using Fractional Derivatives
}

\author{
Antonin Novak
}

\section{To cite this version:}

Antonin Novak. Modeling Viscoelastic Properties of Loudspeaker Suspensions Using Fractional Derivatives. Journal of the Audio Engineering Society, 2016, 64 (1/2), pp.35-44. 10.17743/jaes.2015.0091 . hal-02504339

\section{HAL Id: hal-02504339 \\ https://hal.science/hal-02504339}

Submitted on 10 Mar 2020

HAL is a multi-disciplinary open access archive for the deposit and dissemination of scientific research documents, whether they are published or not. The documents may come from teaching and research institutions in France or abroad, or from public or private research centers.
L'archive ouverte pluridisciplinaire HAL, est destinée au dépôt et à la diffusion de documents scientifiques de niveau recherche, publiés ou non, émanant des établissements d'enseignement et de recherche français ou étrangers, des laboratoires publics ou privés. 


\title{
Modeling Viscoelastic Properties of Loudspeaker Suspensions Using Fractional Derivatives
}

\author{
Antonin Novak* \\ Laboratoire d'Acoustique de I'Université du Mans, LAUM - UMR 6613 CNRS, Le Mans Université, Avenue Olivier \\ Messiaen, 72085 LE MANS CEDEX 9, France \\ *antonin.novak@univ-lemans.fr \\ *https://ant-novak. com
}

\begin{abstract}
A lot of materials used in loudspeaker suspensions exhibit significant frequency dependence of damping and compliance due to their various viscoelastic properties. Few empirical models have been proposed to take into account these variations, the most used one is the LOG model proposed by Knudsen and Jensen. On the other hand, the fractional calculus has been successfully applied to characterize the rheological properties of viscoelastic materials including their frequency dependence. This study shows that the loudspeaker suspension can be successfully modeled using fractional derivatives. The traditional low-frequency loudspeaker model is extended by a simple fractional element. It is shown on four loudspeakers with different types of suspension with different properties that the proposed model fits well with all the studied cases.
\end{abstract}

The archived file is not the final published version of the article A. Novak (2016), "Modeling Viscoelastic Properties of Loudspeaker Suspensions Using Fractional Derivatives", J. Audio Eng. Soc. Vol. 64(1), pp. 35-44.

The definitive publisher-authenticated version is available online at https://doi.org/10.17743/jaes.2015.0091, Readers must contact the publisher for reprint or permission to use the material in any form. 


\section{INTRODUCTION}

A traditional electrodynamic loudspeaker is equipped with a suspension which is usually formed by both a surround attached to the outer edge of the diaphragm and a spider attached to the voice-coil. The purpose of the suspension is to center and adjust the voice-coil in the air gap and to allow a desired axial motion of the diaphragm while preventing lateral motion or rocking. The stiffness of the surround, along with the moving mass, determines the resonance frequency of the loudspeaker and the cone excursion.

Materials used for loudspeaker suspensions have been improved dramatically over the last ten years and will probably keep improving within next decades. While the spider is usually made of impregnated textiles (cotton, poly-cotton, nomex), the surround can be made of different types of materials such as rubber, foam, coated fabric or diaphragm material (rigid mounting). These materials exhibit various viscoelastic properties leading to different loudspeaker characteristics.

It has been shown is several studies $[1,2,3]$ that the traditional model of loudspeaker fails to describe the complicated viscoelastic behavior of these materials. Thus, several different models have been proposed based either on rheological or on empirical approaches [1,2,3]. These models are discussed in section 2.

On the other hand, many physical processes, including the viscoelastic materials, exhibit fractional order behavior [4]. The fractional derivatives, discussed in section 3, provide an adequate description of real viscoelastic behavior with a limited number of material parameters [5]. Moreover, there exists a physical interpretation of the fractional derivatives $[6,7,8,9]$ giving them a full credit over the purely empirical models.

This paper shows how fractional derivatives can provide a suitable method of describing the properties of viscoelastic materials used for loudspeaker suspensions. The aim of the paper is not to evaluate a general theory of fractional derivatives, nor to provide an extensive comparison of viscoelastic models, but to show how to successfully use fractional derivatives for suspension modeling. Adding a single fractional viscoelastic element to the traditional model of loudspeaker, discussed in section 4 , leads to a very good agreement between the measured data and the model. Four loudspeakers, each of them equipped with different types of surround, are used to test the proposed fractional model. The loudspeakers, the experimental setup and the results are discussed in section 5. Moreover, the frequency dependence of the compliance and the mechanical losses is studied in section 6 and a comparison between the measured data and the model based on fractional derivatives is provided. The proposed fractional model provides a very good fit with the measured data in all studied cases.

\section{VISCOELASTIC MODELS OF SUSPENSION}

The materials used for loudspeaker suspensions exhibit both viscous and elastic characteristics. The rheological models describing these characteristics consist of a combination of basic elements such as linear elastic springs (Fig. 1a) and viscous dampers (Fig. 1b) [10]. Simple models, such as Kelvin-Voigt and Maxwell ones, use one spring and one damper connected in parallel and in series respectively. More complicated models consist of more springs and dampers, such as a Standard Linear Solid model (SLS), also known as the Zener model, combining 3 elements [11]. Obviously, the greater the number of springs and dampers, the better the fitting the material behavior of the suspension. The most general viscoelastic models used in rheology, such as Generalized Kelvin-Voigt or the Generalized Maxwell ones, consist of an arbitrary number of elements [11, 12, 13].

\subsection{Traditional model}

The traditional well-known loudspeaker model $[14,15]$ is based on a simple mass-spring-damper system (Fig. 2a). In such a model the mechanical part consists of a mass $m$ attached to a parallel combination of a Newtonian damper defined by its viscous damping coefficient $r$ and a Hookean elastic spring defined by its stiffness $k$. The electro-mechanical analogy to such a system is depicted in (Fig. 2b) in which the mass, damping coefficient (also 


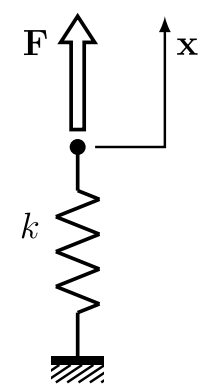

(a)

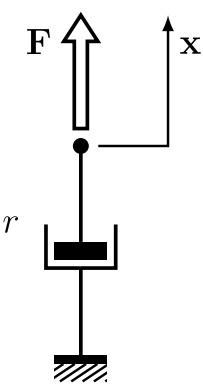

(b)

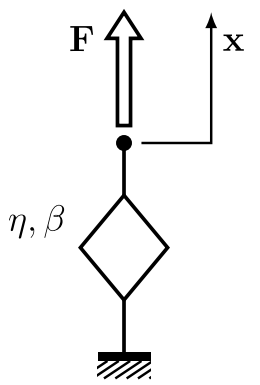

(c)

Figure 1. Basic rheological elements: (a) a purely elastic element = spring, (b) a purely viscous element $=$ damper, (c) a fractional element $=$ interpolation between the spring and the damper.

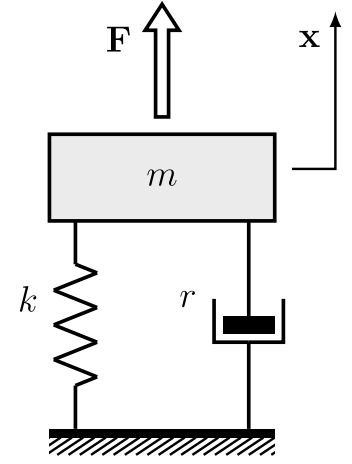

(a)

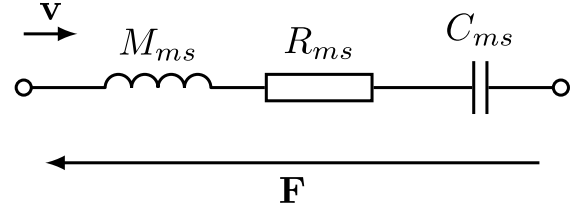

(b)

Figure 2. (a) A traditional mass-spring-damper system based on Kelvin-Voigt viscoelastic model consisting of a Newtonian damper and a Hookean elastic spring connected in parallel. (b) Its electro-mechanical analogy. 
called mechanical resistance) and compliance (the inverse of stiffness) are represented by symbols $M_{m s}, R_{m s}$ and $C_{m s}$ as it is a common practice in the field of audio engineering. Such a model is widely used not only by audio engineers, but also by many scientists most likely due to its low number of parameters and its simplicity.

It is, however, commonly experienced that the traditional 3 parameters model based on a simple mass-springdamper does not describe correctly the real-world behavior of a viscoelastic material. One of the drawbacks of the traditional model is that its mechanical losses are represented purely by viscous damper, omitting possible elastic or viscoelastic losses. Another important drawback is that it does not account for the viscoelastic creep at low frequencies observed in many previous works dealing with loudspeaker suspensions [1, 16, 17]. A natural consequence of such drawbacks is an effort to improve the classical viscoelastic model in such a way that it fits the real-world behavior of a viscoelastic material correctly while still keeping the number of parameters low.

\subsection{Linear Solid models}

A straightforward solution to improve the model is to add spring and damper elements to the Kelvin-Voigt model in series, parallel or in combination of both.

The Standard Linear Solid (SLS) model is a commonly used rheological model for viscoelastic materials. It ameliorates the Kelvin-Voigt model by putting a spring element in series. The study of Knudsen and Jensen [1], in which the spring of the traditional model is replaced by the SLS model, has shown that such a model improves the traditional model. Such a conclusion is not surprising as two extra parameters are added to the model.

More general linear solid models such as the Generalized Maxwell model [12], or parallel connection of so-called three-parameter model [13] may also better fit the material behavior of the suspension, yet at the cost of a greater number of parameters.

\subsection{Models with Frequency Dependent Parameters}

In the study of Knudsen and Jensen [1], two models containing only one extra parameter have been tested: the EXP (exponential) and the LOG (logarithmic) model. Both models use a complex frequency dependent compliance defined as $c_{e}(j \omega)^{-\beta}$ and $c_{l}[1-\lambda \log (j \omega)]$ respectively. According to studies in which the LOG model is compared with the SLS model [17], the LOG model performs much better than the SLS model. A similar conclusion is also found in [1], where the LOG is performing the best.

The LOG model becomes a widely accepted model of complex compliance $[2,3]$ for its simplicity (only 1 extra parameter is needed) and for its sufficiently accurate prediction of the behavior of a viscoelastic material.

However, as the LOG model is purely empirical, and not derived from physical considerations [3], it involves two important drawbacks. First, as the frequency approaches zero, the compliance of the LOG model approaches infinity. Second, above a certain frequency the LOG model predicts negative compliance which is physically impossible $[2,3]$. Moreover, according to [18], the LOG model performs well for suspensions with high degree of viscoelasticity, but fails when the degree of viscoelasticity is low.

In the work of Thorgborg et al. [2], a modified LOG model is proposed including only frequency-dependent damping (FDD). The FDD model is refined in [18] to also include the frequency dependence of compliance (modified FDD). Contrary to the original LOG model, the frequency dependence of damping and the frequency dependence of compliance are separated, involving one extra parameter. Nevertheless, even if the separation of frequency dependencies of damping and compliance may get more physical sense, the model remains still empirical and has the same drawbacks as the LOG model.

\subsection{Models Based on Retardation Spectrum}

Recently, Ritter and Agerkvist [3] found an interesting connection between the empirical LOG model and the Generalized Kelvin-Voigt model (a physical model with infinite number of elements) through the retardation spectrum $[10,19]$. Ritter and Agerkvist proposed two new models (3PC and 4PC). The 3PC model uses three parameters to 
describe the complex frequency dependent compliance and corrects the problem of negative compliance at higher frequencies. The $4 \mathrm{PC}$ model corrects both drawbacks of the LOG model by defining a minimum compliance and an equilibrium compliance. According to authors, the 4PC model, which uses 4 parameters to model the complex compliance, provides slightly better accuracy than the LOG model.

\section{FRACTIONAL VISCOELASTIC ELEMENT}

The use of fractional derivatives, is nowadays accepted in many fields of physics and engineering [4], including mechanics [20], acoustics [21, 22] and more recently audio-engineering [23]. In [23], the fractional derivatives are successfully used to model the electrical impedance of a loudspeaker motor.

The purpose of this paper is not to give a review of fractional derivatives since many information can be found in $[8,24,25]$, but to show that the fractional derivatives can describe better the complex viscoelastic behavior of the loudspeaker suspension and consequently can be successfully used in modeling the loudspeaker mechanical part.

The fractional derivatives are based on the generalization of the differential operator $\frac{d^{n}}{d t^{n}}$, in which $n$ is a positive integer number, to $\frac{d^{\beta}}{d t^{\beta}}$ where $\beta$ is any real number. A typical application of fractional derivatives is a rheological fractional element depicted in Fig. 1c. The force-displacement relation of such an element is given by

$$
\mathbf{F}(t)=\eta \frac{d^{\beta} \mathbf{x}}{d t^{\beta}},
$$

where the constant $\eta$ is called a viscoelastic coefficient, its physical dimension being $\left[\mathrm{Ns}^{\beta} / \mathrm{m}\right][26]$.

Comparing the fractional element with the traditional elements such as spring (Fig. 1a) and damper (Fig. 1b), we conclude that relation (1) can represent the purely elastic element (spring) by putting $\beta=0$ as well as the purely viscous element (damper) by putting $\beta=1$. The fractional element can be seen as an interpolation between the spring and the damper; $\beta \in(0,1)$ indicating the degree between the viscosity and the elasticity. Some authors [27] associate the coefficient $\beta \in(0,0.5)$ to the elasto-viscous materials, and the coefficient $\beta \in(0.5,1)$ to the visco-elastic materials.

\section{APPLICATION OF FRACTIONAL DERIVATIVES TO THE LOUDSPEAKER SUSPENSION}

In this section, the fractional derivatives are applied to the classical mechanical equation of loudspeaker that describes the simple mass-spring-damper system of Fig. 2 by

$$
\mathbf{F}(t)=M_{m s} \frac{d^{2} \mathbf{x}}{d t^{2}}+R_{m s} \frac{d \mathbf{x}}{d t}+\frac{1}{C_{m s}} \mathbf{x}
$$

$\mathbf{F}(t)$ being the force applied to the mechanical part of the loudspeaker and $\mathbf{x}(t)$ being its consecutive displacement.

Adding the fractional element depicted in Fig. 1c in parallel to the traditional model of Fig. 2a, we obtain a new proposed model depicted in Fig. 3. The relation between the force and the displacement can be defined in the time-domain as

$$
\mathbf{F}(t)=M_{m s} \frac{d^{2} \mathbf{x}}{d t^{2}}+R_{v} \frac{d \mathbf{x}}{d t}+\eta \frac{d^{\beta} \mathbf{x}}{d t^{\beta}}+\frac{1}{C_{0}} \mathbf{x}
$$

or in frequency domain, defining $F(\omega)$ and $X(\omega)$ as the Fourier transforms of $\mathbf{F}(t)$ and $\mathbf{x}(t)$, as

$$
\frac{F(\omega)}{X(\omega)}=-\omega^{2} M_{m s}+j \omega R_{v}+(j \omega)^{\beta} \eta+\frac{1}{C_{0}} .
$$

In this modified model $R_{v}$ represents viscous losses and $C_{0}$ represents the static compliance. The mechanical impedance $Z_{m}(\omega)$, defined as the force over the velocity, of such a system is then defined as

$$
Z_{m}(\omega)=j \omega M_{m s}+R_{v}+(j \omega)^{(\beta-1)} \eta+\frac{1}{j \omega C_{0}} .
$$




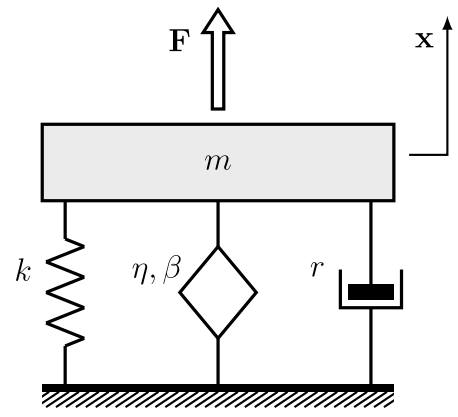

(a)

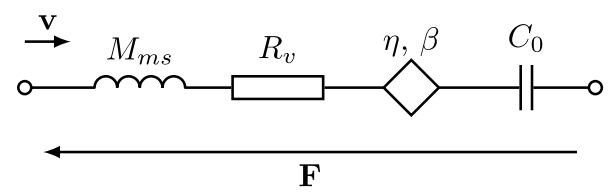

(b)

Figure 3. (a) A proposed mechanical model of the loudspeaker suspension in which a fractional element is added in parallel to the traditional mass-spring-damper system. (b) Its electro-mechanical analogy.
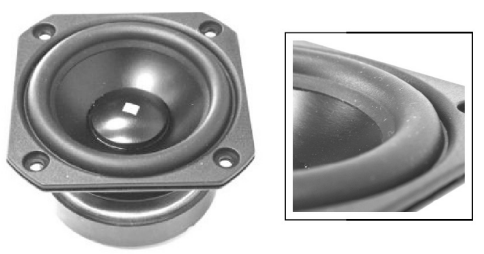

(a)

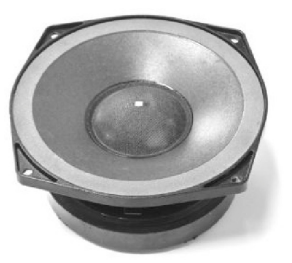

(c)

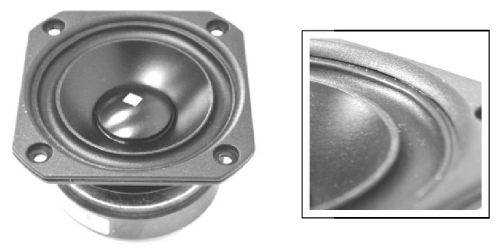

(b)

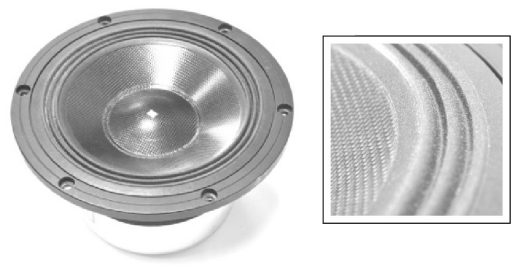

(d)

Figure 4. Evaluated loudspeakers: (a) Peerless PLS-75F25AL02-08, (b) Peerless PLS-75F25AL04-08, (c) PHL 1520, (d) Pioneer W16FU90-51D-T. 


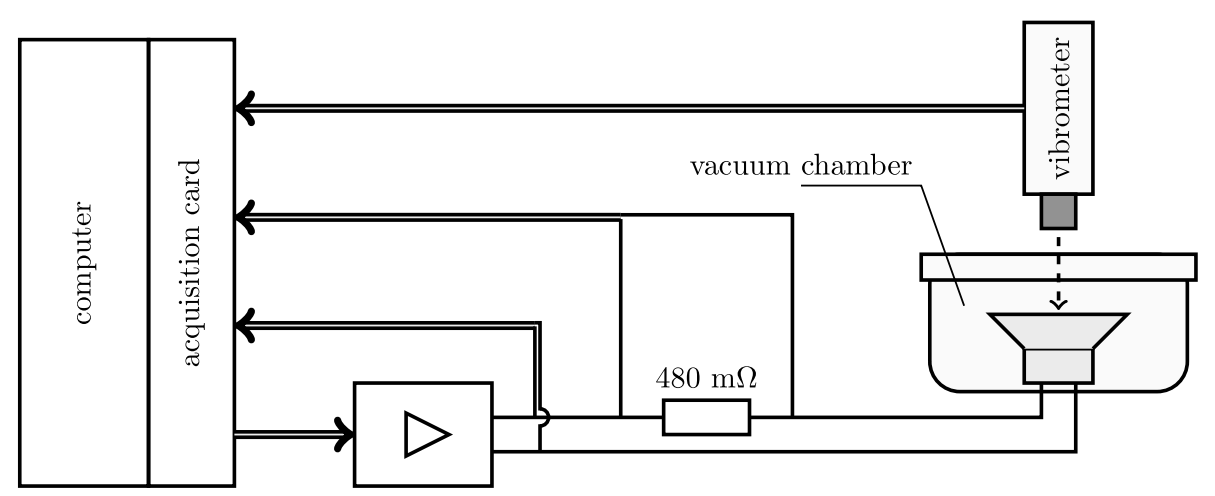

Figure 5. A block diagram of the measuring system. The measurements are performed for two different configurations: under vacuum of -0.97 bar (the vacuum chamber is closed), and in standard atmosphere (the vacuum chamber is opened).

\section{EXPERIMENTS}

In the following, 4 different loudspeakers are evaluated, each of them having a different type of suspension.

Peerless PLS-75F25AL02-08 is a 3" compact full range driver equipped with an anodized aluminium cone, a large roll SBR low-damping rubber surround and a Nomex spider. (Fig. 4a).

Peerless PLS-75F25AL04-08 is almost identical copy of the first loudspeaker (3" full range driver) except that this one is equipped with an inverted rubber surround (Fig. 4b).

PHL 1520 is a 6.5" midrange driver with high-strength cellulose fiber cone impregnated and coated on both sides with damped resins. The suspension consists of a cup spider made of Nomex and a high-speed flat damped surround made of a light-weight strip of polyethylene foam (Fig. 4c).

Pioneer W16FU90-51D-T is a 6.5" woofer with a woven aramid/carbon composite shell cone suspended by a corrugated surround and a cup spider made of Nomex (Fig. 4d).

\subsection{Measurement Set-up}

A block diagram of the measuring system used to both excite the loudspeaker under test and acquire the data is shown in Fig. 5. The loudspeaker is driven with a voltage source (a source having a very low output impedance) as it is usually operated in actual conditions. A NI-4431 USB acquisition device is used to send and acquire the measured signals, and a Devialet D-Premier amplifier to drive the loudspeaker. The current flowing through the loudspeaker is measured using a Current Shunt (N4L HF003) with a resistance of $0.48 \Omega$. The displacement is measured using a Polytec OFV-3000 vibrometer.

Prior to measurements, the loudspeakers are run-in for several hours using a low-frequency high-displacement signal to ensure a mechanical stability during the measurement. Each loudspeaker is put into a small vacuum chamber (Klippel Vacuum Kit) and two series of measurement are made: in standard atmosphere (vacuum chamber opened) and under vacuum of -0.97 bar. In vacuum, no air should influence the motion of mechanical part of the loudspeaker, possibly responsible for a fraction of mechanical losses caused by the viscosity of the air in the gap $[28,29]$. Also, the radiation impedance should be zero. Since the radiation impedance in standard atmosphere and at low frequencies is dominated by a mass loading reactance, it is equivalent to adding a mass proportional to the equivalent surface of the membrane. Thus, the reason to measure the loudspeaker in vacuum and in standard atmosphere is twofold: first to test if the proposed model estimates a similar differences in mass for same size of membranes and, second, to verify if the presence of air influences the mechanical losses. 
Table 1. Estimated mechanical parameters of the model with fractional derivatives for all four loudspeakers measured in standard atmosphere (atm) and in vacuum, and their respective RMS errors (magnitude and phase) of responses from force to displacement $X(\omega) / F(\omega)$.

\begin{tabular}{|c|c|c|c|c|c|c|c|c|}
\hline & \multicolumn{5}{|c|}{ parameters } & \multicolumn{2}{|c|}{ RMS error of $X(\omega) / F(\omega)$} \\
\hline & & $M_{m s}[\mathrm{~g}]$ & $C_{0}[\mathrm{~mm} / \mathrm{N}]$ & $R_{v}[\mathrm{Ns} / \mathrm{m}]$ & $\eta\left[\mathrm{Ns}^{\beta} / \mathrm{m}\right]$ & $\beta[-]$ & mag. [\%] & $\angle[\mathrm{deg}]$ \\
\hline \multirow{2}{*}{ Peerless PLS-75F25AL02-08 } & atm & 2.69 & 1.12 & 0.167 & 140 & 0.140 & 0.22 & 0.13 \\
\hline & vacuum & 2.53 & 1.12 & 0.173 & 140 & 0.141 & 0.26 & 0.17 \\
\hline \multirow{2}{*}{ Peerless PLS-75F25AL04-08 } & atm & 2.52 & 1.20 & 0.182 & 111 & 0.173 & 0.25 & 0.19 \\
\hline & vacuum & 2.37 & 1.18 & 0.190 & 96.6 & 0.194 & 0.31 & 0.21 \\
\hline \multirow{2}{*}{ PHL 1520} & atm & 11.9 & 0.182 & 1.95 & 1889 & 0.146 & 0.51 & 0.38 \\
\hline & vacuum & 10.0 & 0.203 & 2.26 & 2141 & 0.150 & 0.55 & 0.44 \\
\hline \multirow{2}{*}{ Pioneer W16FU90 } & atm & 19.4 & 0.740 & 0.754 & 402 & 0.170 & 0.31 & 0.23 \\
\hline & vacuum & 17.2 & 0.722 & 0.236 & 424 & 0.189 & 0.27 & 0.16 \\
\hline
\end{tabular}

\subsection{Measurement Conditions}

The mechanical properties of the loudspeaker may vary with time due to mechanical fatigue [30, 31], temperature or humidity variation. Therefore, rather then using a sine measurement in which each frequency is applied at a different time, it is preferable to use a multi-tone stimuli where all the frequencies are applied at the same time. The loudspeaker is driven by a multi-tone signal with 80 frequencies logarithmically distributed between $0.1 \mathrm{~Hz}$ and $1 \mathrm{kHz}$. The exact value of each frequency component is adjusted to be a divisor of the sampling frequency $\left(f_{s}=5 \mathrm{kHz}\right)$ of the acquisition system. The phase of each frequency is chosen arbitrarily to avoid a huge crest factor of the input signal.

The choice of the input level is affected by two contradictory facts: it must not be too low, otherwise the signal would be drawn in the noise, but also not too high to overcome the problems associated with nonlinearities of the loudspeaker. A signal with peak value not exceeding $0.2 \mathrm{~V}$ is chosen as a compromise.

The acquired voltage $u(t)$, current $i(t)$ and displacement $x(t)$ are recorded, and the Fourier coefficients of each frequency component $\omega_{i}=2 \pi f_{i}$ of each signal are calculated, resulting in $U\left(\omega_{i}\right), I\left(\omega_{i}\right)$ and $X\left(\omega_{i}\right)$. Approaching the frequency $300 \mathrm{~Hz}$, the assumption of the piston motion of the membrane is not valid due to cone breakup-rim resonances. Since the measured displacement $x(t)$ of the cone is not reliable above this frequency, the measured data above $300 \mathrm{~Hz}$ are ignored.

\subsection{Results}

Force factor $B l$ is first estimated from measured data [32] and the force $F(\omega)$ applied on the mechanical part is calculated as $F(\omega)=B l \cdot I(\omega)$. The receptance frequency response functions [33] $X(\omega) / F(\omega)$ are next derived as well as the mechanical impedance $Z_{m}(\omega)=F(\omega) / V(\omega)$.

To estimate the best set of parameters of the fractional model a curve fitting procedure is performed by minimizing the sum of error (least-squares fit) between $0.1 \mathrm{~Hz}$ and $300 \mathrm{~Hz}$. The fit is performed on both the real and the imaginary part of the mechanical impedance (5), expressed as

$$
\Re\left\{Z_{m}\right\}=R_{v}+\sin \left(\beta \frac{\pi}{2}\right) \omega^{(\beta-1)} \eta,
$$

and

$$
\Im\left\{Z_{m}\right\}=\omega M_{m s}-\cos \left(\beta \frac{\pi}{2}\right) \omega^{(\beta-1)} \eta-\frac{1}{\omega C_{0}} .
$$

The estimated parameters are listed in Table 1, for the four measured loudspeakers, and for both configurations: measured in standard atmosphere, noted as (atm), and measured in vacuum. The respective RMS errors (magnitude and phase) of receptances $X(\omega) / F(\omega)$ are listed in the same Table. The most important conclusions from the estimated parameters are the following.

1) The RMS errors are kept very low for both magnitude and phase for all measured loudspeakers. The error of magnitude is between $0.22 \%$ and $0.55 \%$ and the phase error between 0.14 and 0.44 degrees. A similar conclusion 


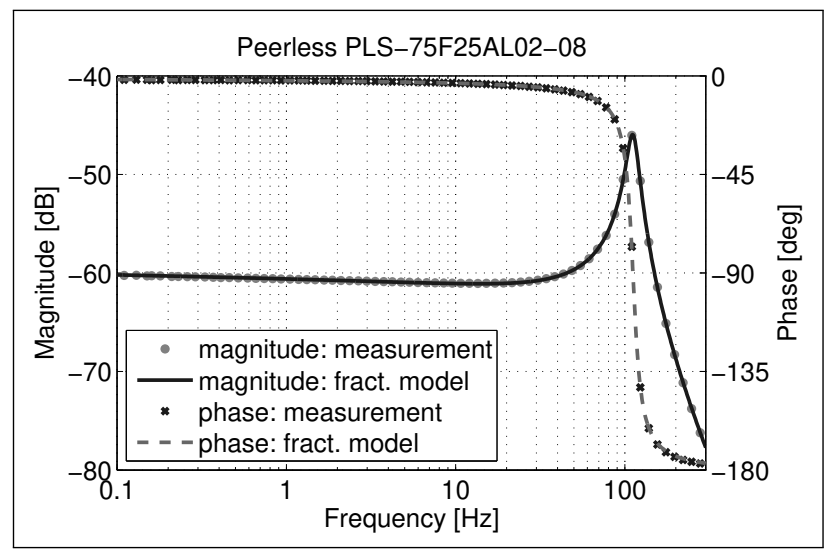

(a)

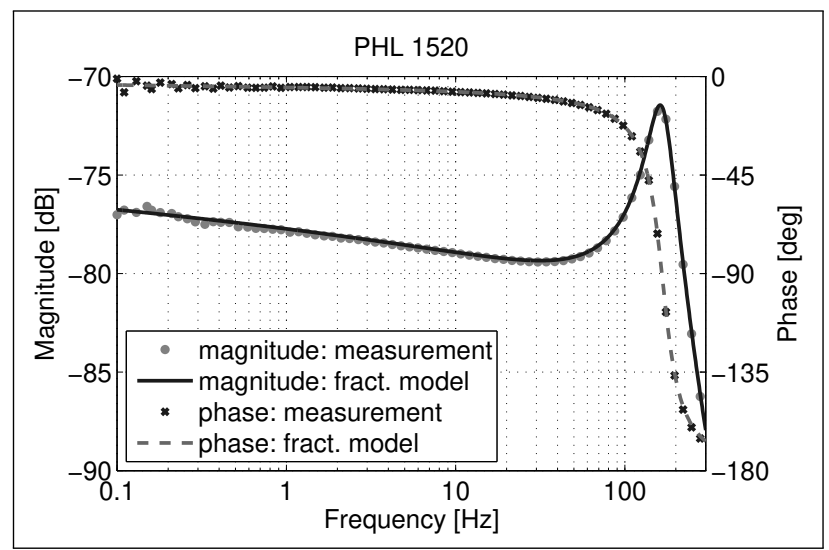

(c)

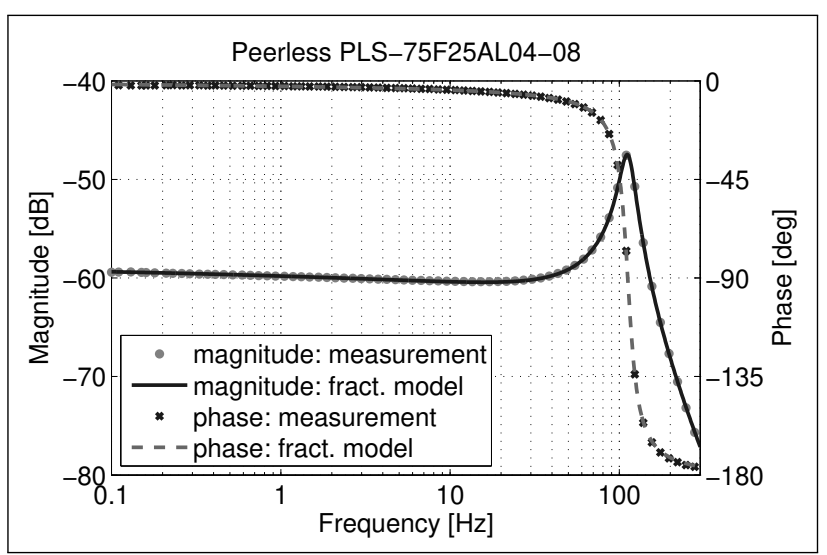

(b)

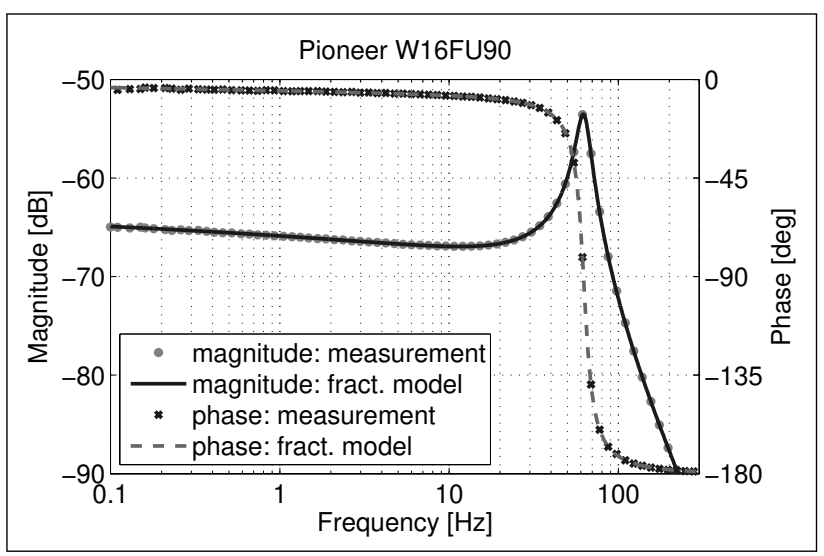

(d)

Figure 6. Measured receptance $X(\omega) / F(\omega)$ of 4 different loudspeakers (dots) compared to a simulation calculated using the model with fractional derivatives. Measurements were performed under vacuum of -0.97 bar. 
can be drawn from Fig. 6 in which the receptances $X(\omega) / F(\omega)$ obtained from measurements in vacuum (dots) are compared to simulated receptances calculated using the model with fractional derivatives. The simulated curves almost perfectly coincide the measured data, even for very low frequencies in which a so-called creep phenomenon appears.

2) The estimated mass $M_{m s}$ is always lower for measurements in vacuum, which agrees with physical laws. Moreover, the mass difference between the measurements in standard atmosphere and in vacuum, corresponding to the air load mass, is $0.16 \mathrm{~g}$ and $0.15 \mathrm{~g}$ in the case of the 3 " loudspeakers and $1.9 \mathrm{~g}$ and $2.2 \mathrm{~g}$ for 6.5 " speakers. The two same-size pairs give almost the same air load mass. Considering the size of equivalent surface of each loudspeaker and their approximative theoretical values of air load [34], the indirectly estimated values of air load mass are reliable.

3) The estimated mechanical parameters $C_{0}, R_{v}, \eta$ and $\beta$ defining the viscoelastic properties of the loudspeaker are almost independent on the atmospheric condition (standard atmosphere or vacuum) for both Peerless speakers and for the PHL speaker. The static compliance $C_{0}$ is almost the same for both Peerless speakers as well as for the Pioneer speaker. A $10 \%$ difference between the measurement of the static compliance $C_{0}$ in standard atmosphere and vacuum is found in the case of the PHL speaker with the foam surround. The viscoelastic parameters $\eta$ and $\beta$ does not vary a lot with the atmospheric condition (standard atmosphere or vacuum). The fractional order $\beta$ always drops a little when changing the atmospheric conditions from vacuum to standard atmosphere but the variation remains very small $(\approx 10 \%)$. The most sensitive parameter to the variation of atmospheric condition is the viscous coefficient $R_{v}$. The viscous coefficient $R_{v}$ of the Pioneer speaker drops by factor of 3 when being in vacuum suggesting a possible losses due to viscosity of the air in the gap, but obviously more scientific proofs would be required to confirm or decline such a conclusion. On the other hand the viscous coefficient $R_{v}$ increases by $4 \%$ for both Peerless speakers and by $15 \%$ for the PHL speaker when being in vacuum. The possible explanation for this variation may be a simple mechanical fatigue [30,31], or a temperature variation influencing the viscoelastic properties of suspension materials. Being aware of a possible decrease of temperature in vacuum, it is measured using a liquid crystal thermometer strip. The drop of temperature is approximately $4 \pm 0.5^{\circ} \mathrm{C}$; from $20 \pm 0.5^{\circ} \mathrm{C}$ in standard atmosphere to $16 \pm 0.5^{\circ} \mathrm{C}$ in vacuum.

4) Finally, viscoelastic coefficient $\beta$ remains low for all four tested loudspeaker, corresponding to rather elastoviscous than visco-elastic behavior [27]. It is also interesting to note that the coefficient $\beta$ is different for both Peerless loudspeakers. These loudspeakers use the same spider and the surround is made of the same rubber material; the only difference is the shape of the surround (a normal surround and an inverted one). The results suggest that the shape of the surround may also influence the model parameters.

\section{FREQUENCY DEPENDENT COMPLIANCE AND LOSSES}

It has been shown in many papers dealing with viscoelastic behavior of loudspeaker suspensions $[1,3,18]$ that the compliance as well as the mechanical losses are frequency dependent. The frequency dependent complex compliance [1] can be written as $C^{*}(\omega)=C^{\prime}(\omega)-j C^{\prime \prime}(\omega)$ [10], where the real-part component $C^{\prime}(\omega)$ is called the storage compliance and determines the storage energy, whilst the imaginary-part component $C^{\prime \prime}(\omega)$ is responsible for energy losses and is consequently called loss compliance.

\subsection{Storage Compliance}

The complex compliance [10,35] of a mechanical system can be expressed as [3]

$$
C^{*}(\omega)=\left[\frac{F(\omega)}{X(\omega)}+\omega^{2} M_{m s}\right]^{-1}
$$

where all the viscous and viscoelastic losses are included in the imaginary part of the complex compliance $C^{*}(\omega)$. 
The complex compliance of the proposed fractional model can be thus derived from (4) and (8) as

$$
C^{*}(\omega)=\left[\frac{1}{C_{0}}+(j \omega)^{\beta} \eta+j \omega R_{v}\right]^{-1} .
$$

Studying closely the storage compliance $C^{\prime}(\omega)$, defined as a real part of (9), we can note, that the fractional order model does not suffer from the drawbacks of the LOG model discussed in section 2.

First, as the frequency approaches zero, the storage compliance approaches the static compliance $C_{0}$. Second, contrary to the LOG model, the storage compliance never reaches negative values. In Fig. 7 the storage compliance derived from measured data in vacuum using (8) is compared with the simulated storage compliance calculated using the fractional model (9). The comparison in Fig. 7 is provided for all the tested loudspeakers except the Peerless with inverted surround since its estimated mechanical parameters, and consequently the storage compliance, are very similar to the Peerless loudspeaker with normal surround and the very close curves would worsen the readability of the graph. We can conclude from Fig. 7 that a good estimation of all the parameters of the proposed fractional model is sufficient to describe correctly the frequency dependent storage compliance within the frequency range of interest.

When comparing the behavior of the storage compliance (Fig. 7) at very low frequencies, we can see that the measured data as well as the fractional model approaches the static compliance $C_{0}$ (see Table 1 ) as frequency tends to zero. However, all tested loudspeaker still exhibit an increase of storage compliance with decreasing frequency even for frequencies approaching $0.1 \mathrm{~Hz}$. Therefore, it still remains unclear at which (very low) frequency the compliance stabilizes to the value of static compliance $C_{0}$.

\subsection{Mechanical Losses}

When studying the mechanical properties of a loudspeaker, the mechanical losses are usually defined as the real part of the mechanical impedance. In a traditional model of loudspeaker (2), the only mechanical losses taken into account are the viscous ones, represented by the viscous coefficient $R_{m s}$. The mechanical losses of the proposed fractional model have two components (6): a constant $R_{v}$ responsible for the viscous losses and a component with frequency dependence $\omega^{(\beta-1)}$ representing the losses caused by the viscoelastic fractional element.

In Fig. 8, the mechanical losses derived from measured data in vacuum as a real part of the measured mechanical impedance $Z_{m}(\omega)$ are compared with the mechanical losses calculated using the fractional model (6). Once again, we can conclude from Fig. 8 that the fractional model gives a very good fit between the measured data and the data calculated using the fractional model.

The two dashed asymptotic lines in Fig. 8 show the relative contribution of the viscous losses (parameter $R_{v}$ ) and viscoelastic losses (parameters $\beta$ and $\eta$ ) for the Pioneer speaker. Note, that the viscous contribution $\left(R_{v}\right)$, usually used as a single parameter to represent losses in the traditional model, is not frequency dependent and can hardly fit the measured decreasing curve. The viscoelastic contribution is represented by a straight line in a log-log graph; the slope being given by $(\beta-1)$.

\subsection{Loss factor}

It is sometimes convenient to define a loss factor $\tan \delta[35]$ relating the storage and the loss energy by the following relation

$$
C^{*}(\omega)=C^{\prime}(\omega)[1-j \tan \delta(\omega)]
$$

where

$$
\tan \delta=\frac{C^{\prime \prime}(\omega)}{C^{\prime}(\omega)}
$$


The loss factor of the fractional model can be thus derived from (9) and (11) as

$$
\tan \delta=\frac{\omega R_{v}+\eta \sin \left(\beta \frac{\pi}{2}\right) \omega^{\beta}}{\frac{1}{C_{0}}+\eta \cos \left(\beta \frac{\pi}{2}\right) \omega^{\beta}} .
$$

In Fig. 9, the loss factors derived from measured data in vacuum (11) are compared with the loss factors calculated using the fractional model (12). The asymptotic behavior due to viscous and viscoelastic contributions are depicted using dashed lines. Note that the slope of the dashed line of the viscous contribution in the log-log plot is equal to one no matter the coefficients of the model ( $\tan \delta=R_{v} C_{0} \omega$ for $\eta=0$ ). Thus, considering only viscous contribution as a single element responsible for the losses would not be sufficient especially at low frequencies where the slope of the $\log -\log$ curve of $\tan \delta$ is much lower than one.

\section{DISCUSSION}

\subsection{Frequency range}

The results of four studied loudspeakers with different types of suspension show that the proposed model with a single fractional element provides very low rms error within the frequency range in which the measured data are reliable. Since the assumption of the piston motion of the membrane is not valid at higher frequencies due to cone breakup-rim resonances, usually occurring two or three octaves above the resonance frequency, the model accuracy cannot be estimated above these frequencies. However, as shown in Figs. 8 and 9 the viscoelastic contribution of the model is much more important under the resonance frequency. Consequently, the frequency limit of the model due to the cone breakup-rim resonances is the same as for the traditional lumped model of an electrodynamic loudspeaker.

\subsection{Number of parameters}

The proposed model uses four parameters to describe the viscoelastic behavior of the mechanical part: the static compliance $C_{0}$ defining the purely elastic properties of the suspension, the viscous coefficient $R_{v}$ defining purely viscous properties, the viscoelastic coefficient $\eta$ and the fractional order $\beta$. More complicated models such as Kelvin-Voigt chain model [36], or Maxwell chain model [36, 37, 38] use more fractional elements to fit better the viscoelastic behavior of the material, but at the cost of greater number of parameters. Lower number of parameters would also be possible, but at the cost of the model precision. Keeping only the fractional element with the spring (putting $R_{v}=0$ ) would result in a straight line of the log-log plot of mechanical losses (6) in Fig 8. Such a choice would lead to an incorrect fit of mechanical losses and consequently to higher error of the model. Keeping only the fractional element with the damper (putting $C_{0}=\infty$ ) would bring the same drawback as the one with the LOG model. Moreover, such a solution has already been proposed by Knudsen and Jensen [1], since the EXP model is indeed a particular case of the proposed fractional model with $C_{0}=\infty$.

\subsection{Nonlinear fractional model}

The proposed fractional model can be applied in the nonlinear domain in several manners. A simple engineering approach would be to make vary at least one of the model parameters $R_{v}, \eta, \beta$ and $C_{0}$ with displacement. More general approach would consist in using a nonlinear model such as Volterra model [39] or Generalized Hammerstein model [40] in which the fractional derivatives would appear inside the nonlinear kernels.

The linear relation between the force and the displacement (3) can be expressed as

$$
\mathbf{F}(t)-M_{m s} \frac{d^{2} \mathbf{x}}{d t^{2}}=\hat{\mathbf{S}}[\mathbf{x}(t)],
$$

where $\hat{\mathbf{S}}$ represents a linear operator defined as

$$
\hat{\mathbf{S}}[\mathbf{x}(t)]=\left[R_{v} \frac{d}{d t}+\eta \frac{d^{\beta}}{d t^{\beta}}+\frac{1}{C_{0}}\right] \mathbf{x} .
$$




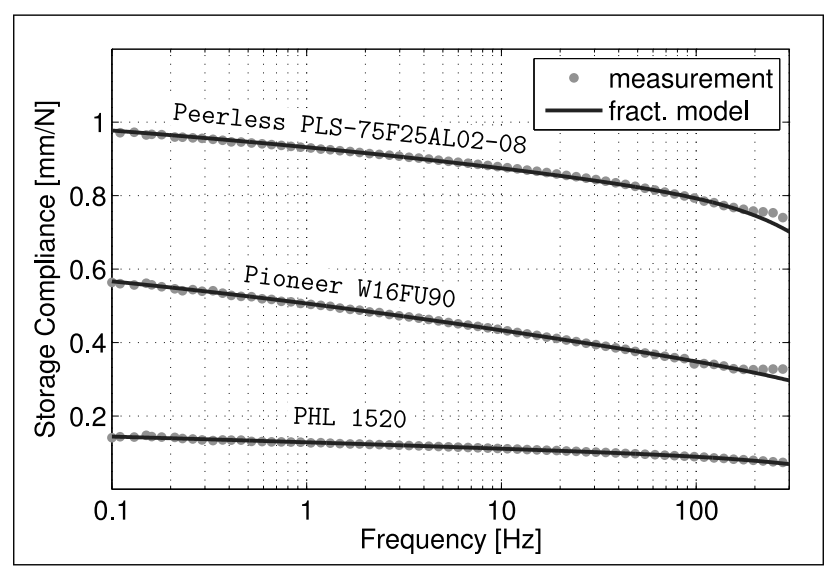

Figure 7. Storage compliance of 3 different loudspeakers derived from the measurement (dots) compared to a simulation calculated using the model with fractional derivatives. Measurements were performed under vacuum of -0.97 bar.

Since the operator $\mathbf{S}$ is linear and translation invariant, it can be represented as a convolution

$$
\hat{\mathbf{S}}[\mathbf{x}(t)]=\mathbf{h}(t) * \mathbf{x}(t),
$$

where the kernel $\mathbf{h}(t)$ is defined using inverse Fourier transform $\mathcal{F}^{-1}$ as

$$
\mathbf{h}(t)=\mathcal{F}^{-1}\left[j \omega R_{v}+\eta(j \omega)^{\beta}+\frac{1}{C_{0}}\right] .
$$

In a case of a nonlinear system, the linear operator $\hat{\mathbf{S}}$ must be replaced by a nonlinear operator $\hat{\mathbf{S}}_{N L}$. Using the Generalized Hammerstein model, the $\hat{\mathbf{S}}_{N L}$ expands to

$$
\hat{\mathbf{S}}_{N L}[\mathbf{x}]=\sum_{n=1}^{N} \mathbf{h}_{n}(t) * \mathbf{x}^{n}(t) .
$$

The fractional derivatives may thus apply not only to the linear kernel $\mathbf{h}_{1}(t)$ (16), but to all nonlinear kernels $\mathbf{h}_{n}(t)$.

Moreover, taking into account a possible hysteresis-like dependence on the peak value of displacement $x_{\max }$ [41], the nonlinear operator $\hat{\mathbf{S}}_{N L}$ should be implemented as a function o instantaneous and peak displacement leading to much more complicated models [42] that require advanced identification techniques of nonlinear systems [43].

\section{CONCLUSION}

This paper shows that the mechanical models based on fractional derivatives may prove useful for loudspeaker suspensions. In view of their ability to model viscoelastic phenomena, they provide a suitable method of describing dynamical properties of viscoelastic materials used in loudspeaker manufacture.

The results of four studied loudspeakers with different types of suspension show that the proposed model with a single fractional element provides very low rms error when compared to the measured data for all loudspeakers measured in standard atmosphere as well as in vacuum. 


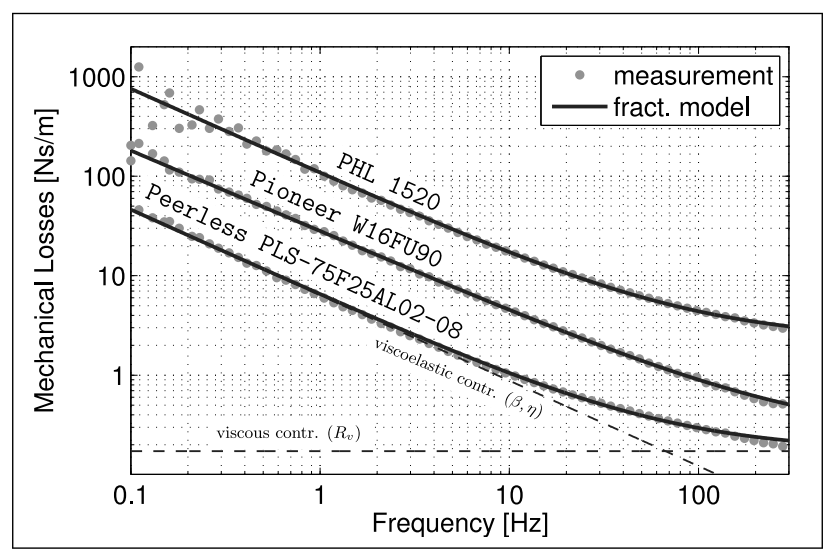

Figure 8. Mechanical losses of 3 different loudspeakers derived from the measurement (dots) compared to a simulation calculated using the model with fractional derivatives. Measurements were performed under vacuum of -0.97 bar. The relative contribution of the viscoelastic losses $(\beta, \eta)$ and viscous losses $\left(R_{v}\right)$ of the Peerless loudspeaker are depicted as dashed asymptotes.

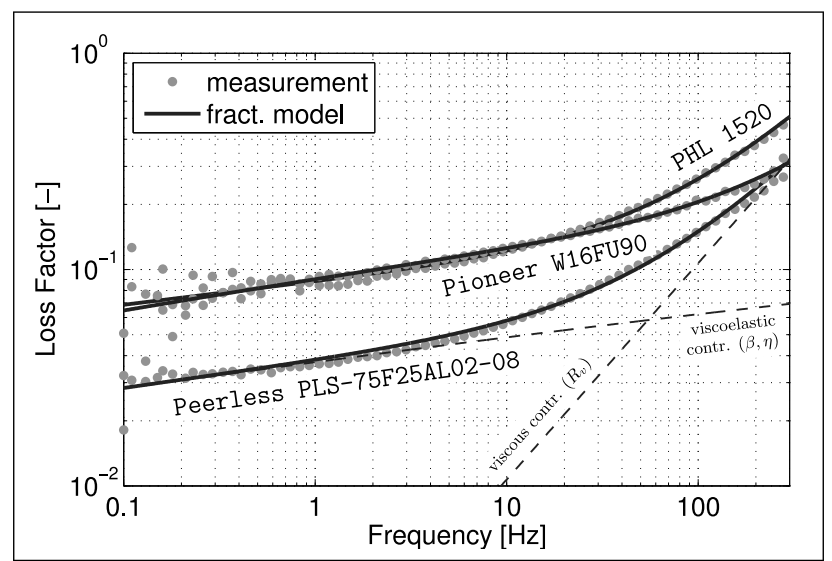

Figure 9. Loss factor $\tan \delta$ of 3 different loudspeakers derived from the measurement (dots) compared to a simulation calculated using the model with fractional derivatives. Measurements were performed under vacuum of -0.97 bar. The relative contribution of the viscoelastic losses $(\beta, \eta)$ and viscous losses $\left(R_{v}\right)$ of the Peerless loudspeaker are depicted as dashed asymptotes. 


\section{References}

[1] M. H. Knudsen and J. G. Jensen, "Low-frequency loudspeaker models that include suspension creep," J. Audio Eng. Soc, vol. 41, no. 1/2, pp. 3-18, 1993.

[2] K. Thorborg, C. Tinggaard, F. Agerkvist, and C. Futtrup, "Frequency dependence of damping and compliance in loudspeaker suspensions," J. Audio Eng. Soc, vol. 58, no. 6, pp. 472-486, 2010.

[3] T. Ritter and F. T. Agerkvist, "Modeling viscoelasticity of loudspeaker suspensions using retardation spectra," in Audio Engineering Society Convention 129, Nov 2010.

[4] J. Sabatier, O. P. Agrawal, and J. T. Machado, Advances in fractional calculus, vol. 4. Springer, 2007.

[5] N. Heymans and J.-C. Bauwens, "Fractal rheological models and fractional differential equations for viscoelastic behavior," Rheologica Acta, vol. 33, no. 3, pp. 210-219, 1994.

[6] H. Schiessel and A. Blumen, "Hierarchical analogues to fractional relaxation equations," Journal of Physics A: Mathematical and General, vol. 26, no. 19, p. 5057, 1993.

[7] H. Schiessel, R. Metzler, A. Blumen, and T. Nonnenmacher, "Generalized viscoelastic models: their fractional equations with solutions," Journal of physics A: Mathematical and General, vol. 28, no. 23, p. 6567, 1995.

[8] R. Hilfer, P. Butzer, U. Westphal, J. Douglas, W. Schneider, G. Zaslavsky, T. Nonnemacher, A. Blumen, and B. West, Applications of fractional calculus in physics, vol. 128. World Scientific, 2000.

[9] K. D. Papoulia, V. P. Panoskaltsis, N. V. Kurup, and I. Korovajchuk, "Rheological representation of fractional order viscoelastic material models," Rheologica acta, vol. 49, no. 4, pp. 381-400, 2010.

[10] J. Ferry, Viscoelastic Properties of Polymers. Wiley, 1980.

[11] G. E. Mase, Schaum's outline of theory and problems of continuum mechanics. McGraw-Hill New York, 1970.

[12] A. Petosic, I. Djurek, and D. Djurek, "Modeling of an electrodynamic loudspeaker including membrane viscoelasticy," in Audio Engineering Society Convention 124, May 2008.

[13] I. Djurek, A. Petosic, and D. Djurek, "Analysis of viscoelasticity and residual strains in an electrodynamic loudspeaker," in Audio Engineering Society Convention 126, Audio Engineering Society, May 2009.

[14] N. Thiele, "Loudspeakers in vented boxes: Part 1," Journal of the Audio Engineering Society, vol. 19, no. 5, pp. 382-392, 1971.

[15] R. H. Small, "Vented-box loudspeaker systems-part 2: Large-signal analysis," Journal of the Audio Engineering Society, vol. 21, no. 6, pp. 438-444, 1973.

[16] M. H. Knudsen, P. Hansen, and J. G. Jensen, "The significance of viscoelastic effects in loudspeakers parameter measurements," in Audio Engineering Society Convention 88, Audio Engineering Society, Mar 1990.

[17] F. Agerkvist, K. Thorborg, and C. Tinggard, "A study of the creep effect in loudspeaker suspension," in Audio Engineering Society Convention 125, Audio Engineering Society, Oct 2008.

[18] K. Thorborg and C. Futtrup, "Frequency dependence of the loudspeaker suspension (a follow up)," J. Audio Eng. Soc, vol. 61, no. 10, pp. 778-786, 2013. 
[19] M. Jirásek and P. Havlásek, "Accurate approximations of concrete creep compliance functions based on continuous retardation spectra," Computers $\mathscr{E}$ Structures, vol. 135, pp. 155-168, 2014.

[20] R. Koeller, "A theory relating creep and relaxation for linear materials with memory," Journal of Applied Mechanics, vol. 77, no. 3, p. 031008, 2010.

[21] S. Holm and R. Sinkus, "A unifying fractional wave equation for compressional and shear waves," The Journal of the Acoustical Society of America, vol. 127, no. 1, pp. 542-548, 2010.

[22] S. Holm and S. P. Näsholm, "A causal and fractional all-frequency wave equation for lossy media," The Journal of the Acoustical Society of America, vol. 130, no. 4, pp. 2195-2202, 2011.

[23] P. Brunet and B. Shafai, "Identification of loudspeakers using fractional derivatives," Journal of the Audio Engineering Society, vol. 62, no. 7/8, pp. 505-515, 2014.

[24] I. Podlubny, Fractional differential equations: an introduction to fractional derivatives, fractional differential equations, to methods of their solution and some of their applications, vol. 198. Academic press, 1998.

[25] A. B. Malinowska, T. Odzijewicz, and D. F. Torres, Advanced Methods in the Fractional Calculus of Variations. Springer, 2015.

[26] W. Grzesikiewicz, A. Wakulicz, and A. Zbiciak, "Non-linear problems of fractional calculus in modeling of mechanical systems," International Journal of Mechanical Sciences, vol. 70, pp. 90-98, 2013.

[27] M. Di Paola and M. Zingales, "Exact mechanical models of fractional hereditary materials," Journal of Rheology (1978-present), vol. 56, no. 5, pp. 983-1004, 2012.

[28] H. Kuttruff, Acoustics: An Introduction. CRC Press, 2007.

[29] A. Voishvillo, "Identification compression driver parameters based on a concept of diaphragm's frequencydependent area," in Audio Engineering Society Convention 137, Audio Engineering Society, Oct 2014.

[30] F. Agerkvist and B. R. Pedersen, "Time variance of the suspension nonlinearity," in Audio Engineering Society Convention 125, Oct 2008.

[31] W. Klippel, "Mechanical fatigue and load-induced aging of loudspeaker suspension," in Audio Engineering Society Convention 131, Audio Engineering Society, Oct 2011.

[32] A. Novak, P. Lotton, and L. Simon, "Nonlinear force factor measurement of an electrodynamic loudspeaker," in Forum Acusticum 2014, Sep 2014.

[33] K. G. McConnell, Vibration testing: theory and practice. John Wiley \& Sons, 1995.

[34] J. Vanderkooy, "The inertial air load of a loudspeaker diaphragm," in Audio Engineering Society Convention 124, Audio Engineering Society, May 2008.

[35] M. Rousseau and J. Vanderkooy, "Visco-elastic aspects of loudspeaker drivers," in Audio Engineering Society Convention 118, May 2005.

[36] R. Koeller, "Applications of fractional calculus to the theory of viscoelasticity," Journal of Applied Mechanics, vol. 51, no. 2, pp. 299-307, 1984. 
[37] A. Hernandez-Jimenez, J. Hernandez-Santiago, A. Macias-Garcı, and J. Sanchez-Gonzalez, "Relaxation modulus in pmma and ptfe fitting by fractional maxwell model," Polymer Testing, vol. 21, no. 3, pp. 325-331, 2002.

[38] Y. Yin and K.-Q. Zhu, "Oscillating flow of a viscoelastic fluid in a pipe with the fractional maxwell model," Applied mathematics and computation, vol. 173, no. 1, pp. 231-242, 2006.

[39] M. Schetzen, The Volterra and Wiener Theories of Nonlinear Systems. New York: John Wiley \& Sons, 1980.

[40] A. Novak, L. Simon, F. Kadlec, and P. Lotton, "Nonlinear system identification using exponential swept-sine signal," Instrumentation and Measurement, IEEE Transactions on, vol. 59, no. 8, pp. 2220-2229, 2010.

[41] A. Novak, P. Lotton, and L. Simon, "Dynamic measurement of loudspeaker suspension parameters using an active harmonic control technique," in Audio Engineering Society Convention 136, Apr 2014.

[42] B. Maillou, Identification et caractérisation des non-linéarités de systèmes acoustiques. PhD thesis, LAUM, University of Maine, France, 2015.

[43] A. Novak, B. Maillou, P. Lotton, and L. Simon, "Nonparametric identification of nonlinear systems in series," Instrumentation and Measurement, IEEE Transactions on, vol. 63, no. 8, pp. 2044-2051, 2014. 